\title{
A Push-Button Molecular Switch
}

\author{
Jason M. Spruell, ${ }^{\dagger}$ Walter F. Paxton, ${ }^{\dagger}$ John-Carl Olsen, ${ }^{\dagger}$ Diego Benítez, ${ }^{\dagger}$ \\ Ekaterina Tkatchouk, ${ }^{\dagger}$ Charlotte L. Stern, ${ }^{\dagger}$ Ali Trabolsi, ${ }^{\dagger}$ Douglas C. Friedman, ${ }^{\dagger}$ \\ William A. Goddard III, ${ }^{\star}$ and J. Fraser Stoddart ${ }^{*}$, \\ Department of Chemistry, Northwestern University, \\ 2145 Sheridan Road, Evanston, IL 60202 and Materials and Process Simulation Center, \\ California Institute of Technology, Pasadena, CA 91125
}

\section{Supporting Information}

\begin{tabular}{|c|}
\hline Correspondence Address \\
\hline Professor J Fraser Stoddart \\
Department of Chemistry \\
Northwestern University \\
2145 Sheridan Road \\
Evanston, IL 60208 (USA) \\
Tel: (+1)-847-491-3793 \\
Fax: (+1)-847-491-1009 \\
Email: stoddart@northwestern.edu \\
\hline
\end{tabular}

†Northwestern University

California Institute of Technology 


\section{Collection of 1D, 2D and VT NMR Spectroscopic Data}
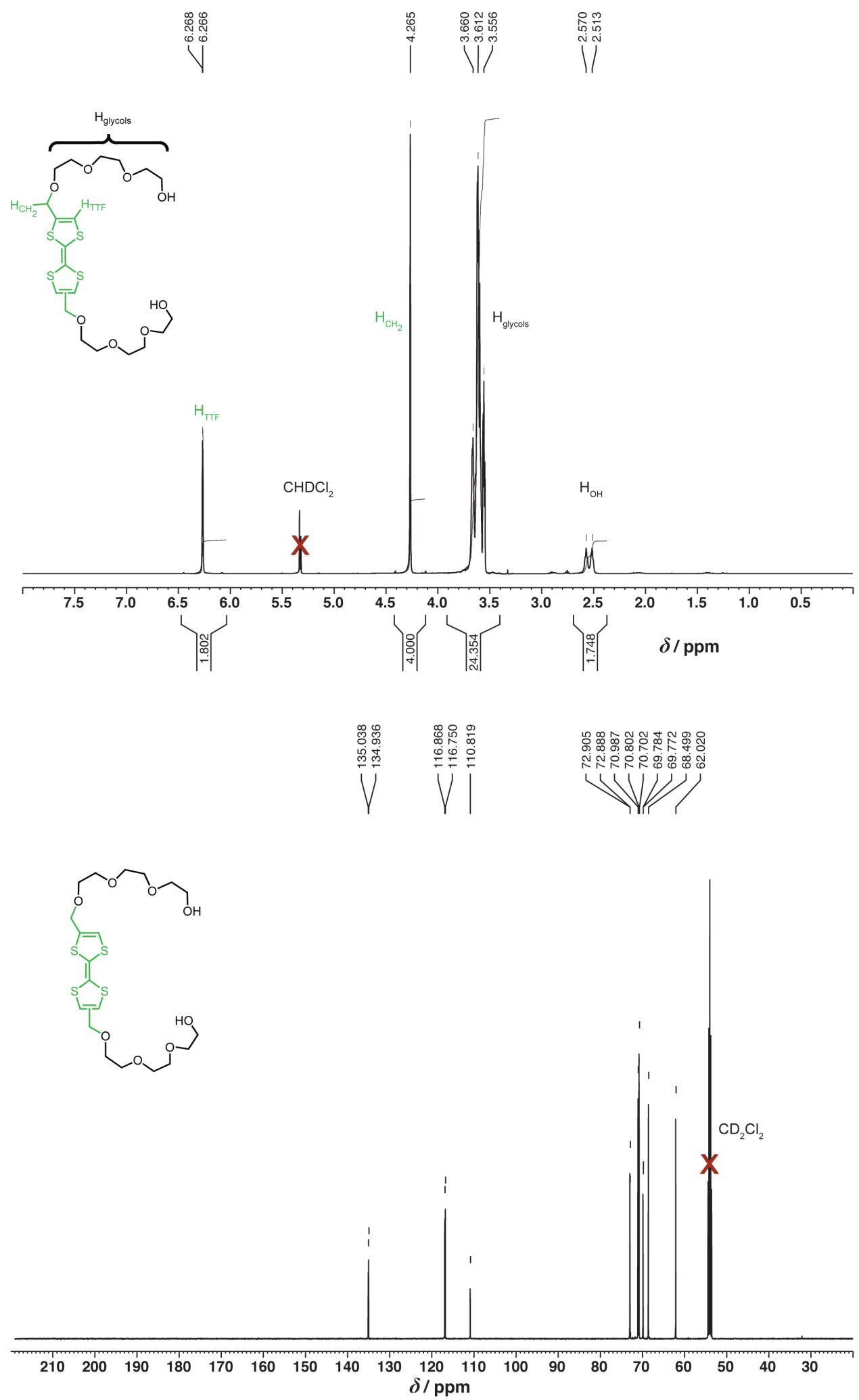

Figure S1: ${ }^{1} \mathrm{H}$ and ${ }^{13} \mathrm{C}$ NMR spectra of $3\left(500 \mathrm{MHz} ; 125 \mathrm{MHz}, 293 \mathrm{~K}, \mathrm{CD}_{2} \mathrm{Cl}_{2}\right)$ 

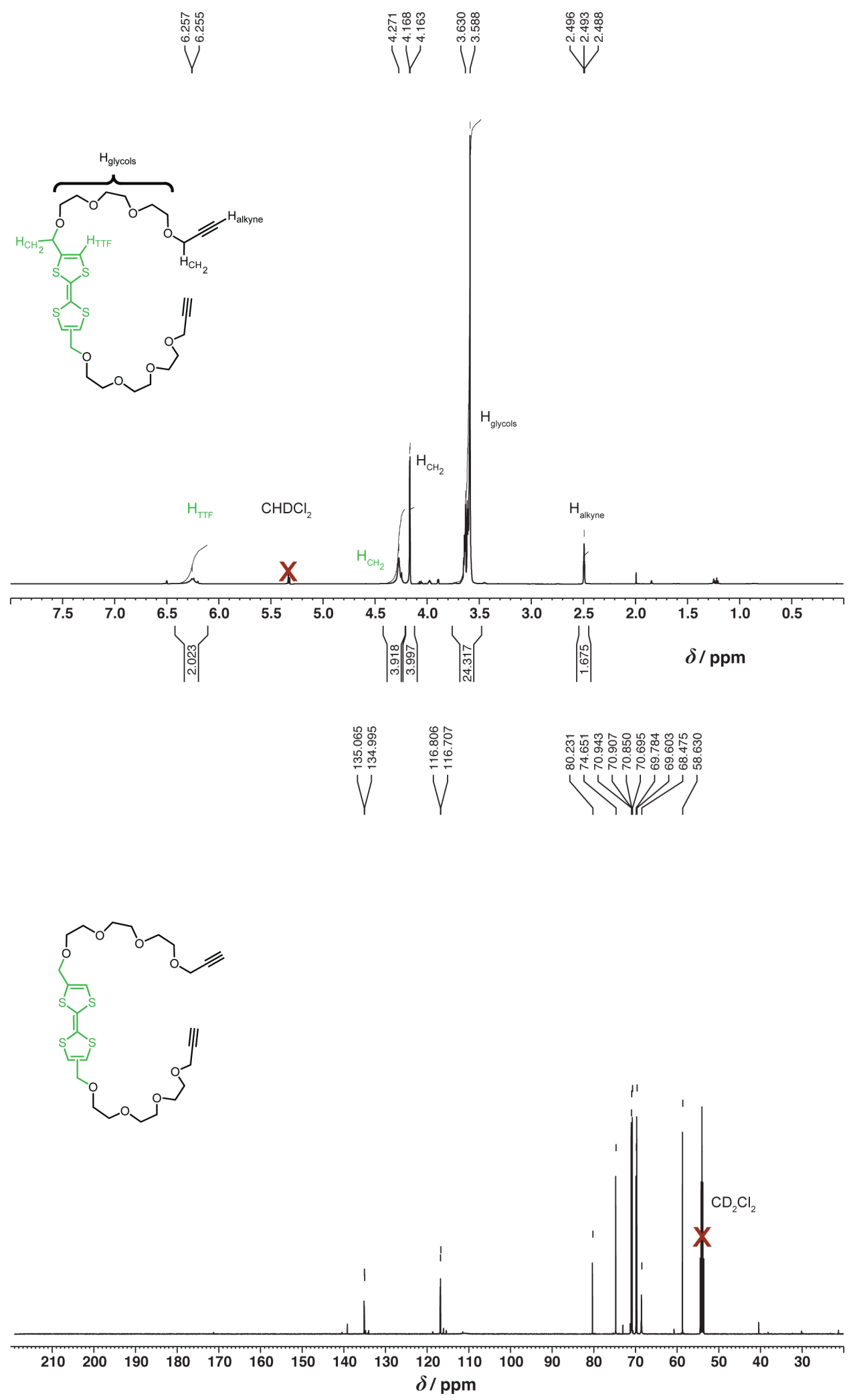

Figure S2: ${ }^{1} \mathrm{H}$ and ${ }^{13} \mathrm{C}$ NMR spectra of $4\left(500 \mathrm{MHz} ; 125 \mathrm{MHz}, 293 \mathrm{~K}, \mathrm{CD}_{2} \mathrm{Cl}_{2}\right)$ 


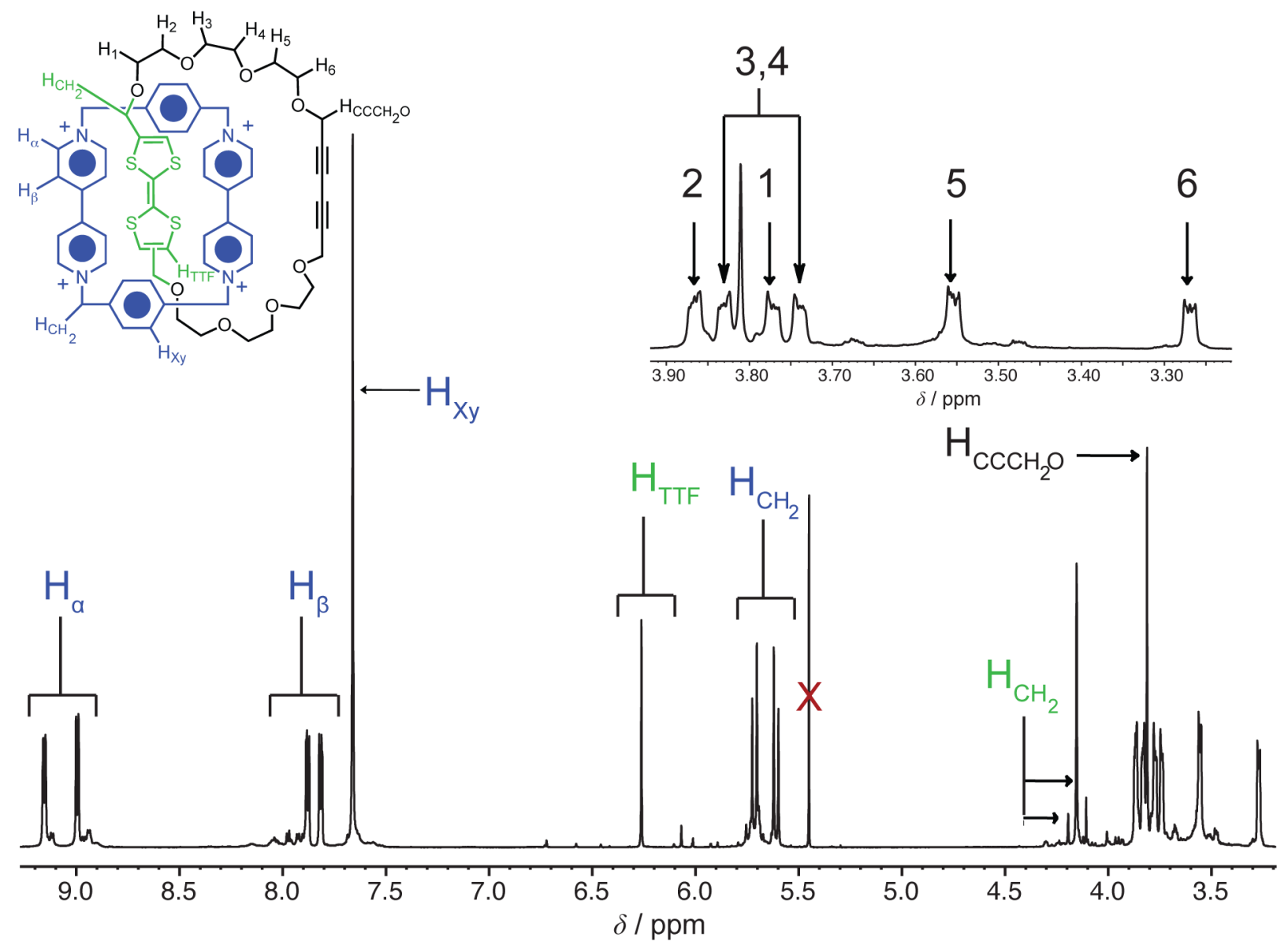

Figure S3: Fully annotated ${ }^{1} \mathrm{H}$ NMR spectrum of $1 \cdot 4 \mathrm{PF}_{6}\left(500 \mathrm{MHz}, 293 \mathrm{~K}, \mathrm{CD}_{3} \mathrm{CN}\right)$ 


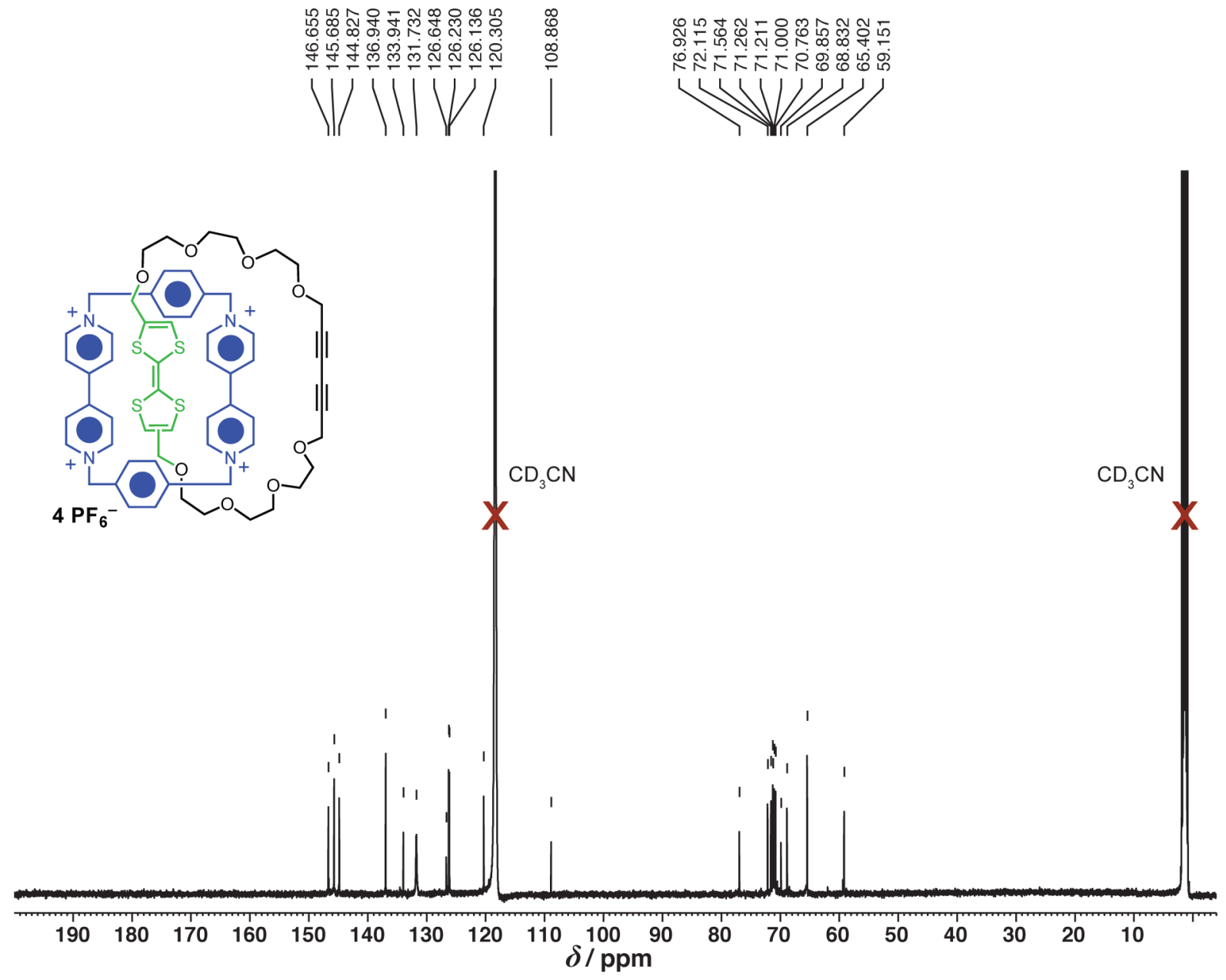

Figure S4: ${ }^{13} \mathrm{C}$ NMR spectrum of $1 \cdot 4 \mathrm{PF}_{6}\left(125 \mathrm{MHz}, 293 \mathrm{~K}, \mathrm{CD}_{3} \mathrm{CN}\right)$ 


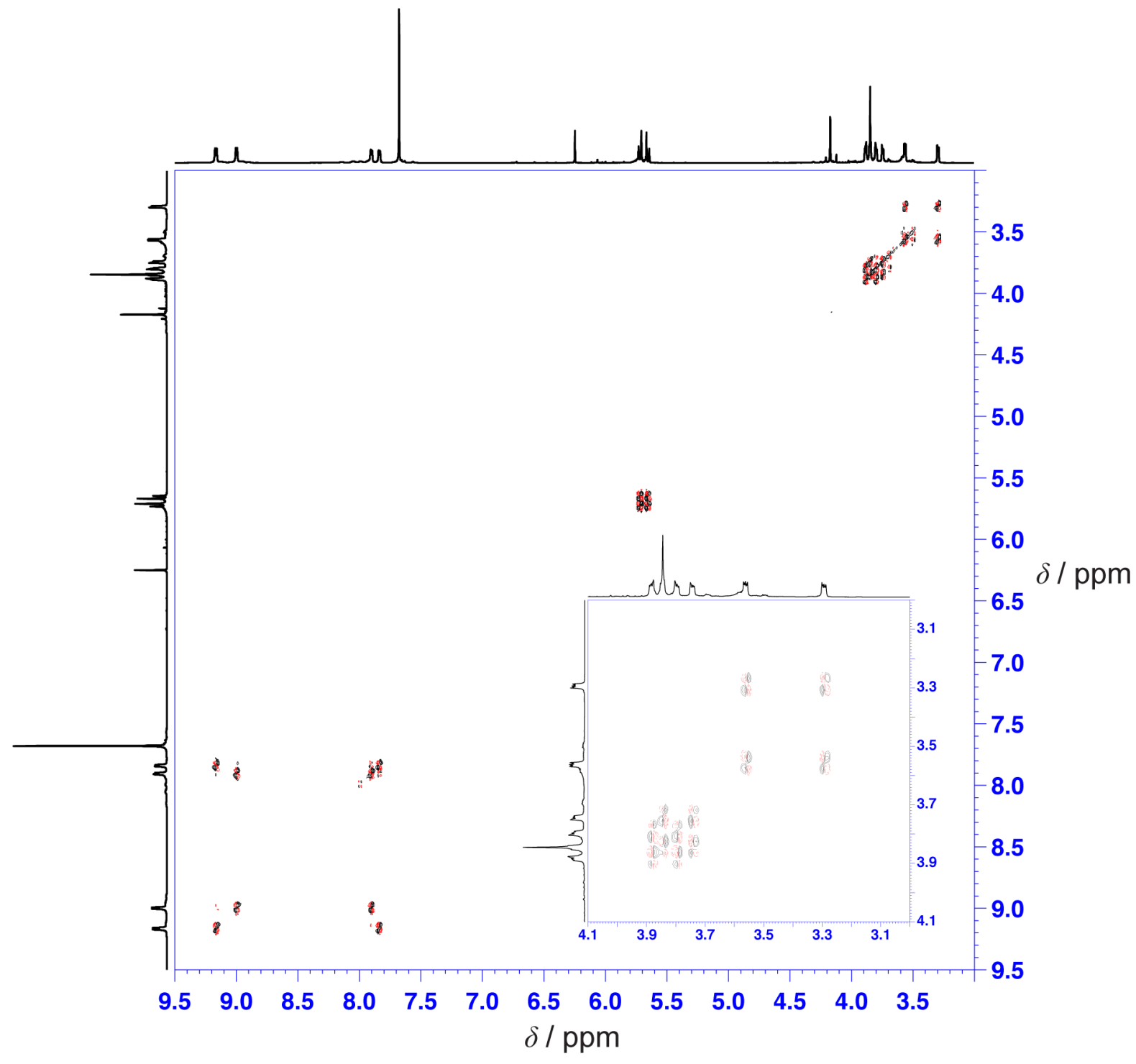

Figure S5: COSY Spectrum of 1·4PF $6(600 \mathrm{MHz}, 293 \mathrm{~K})$ 


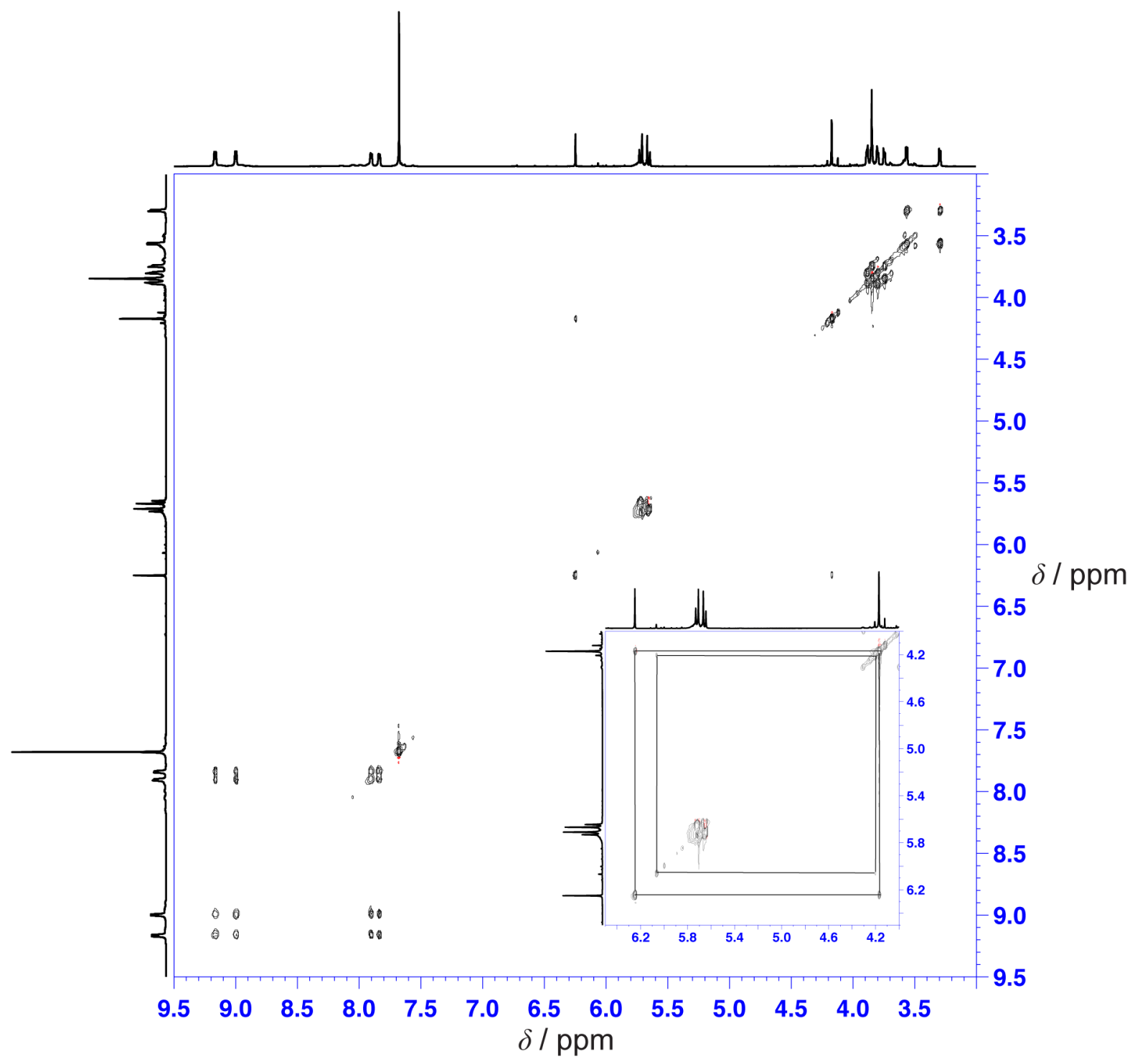

Figure S6: TOCSY Spectrum of 1·4PF $6(600 \mathrm{MHz}, 293 \mathrm{~K})$ 


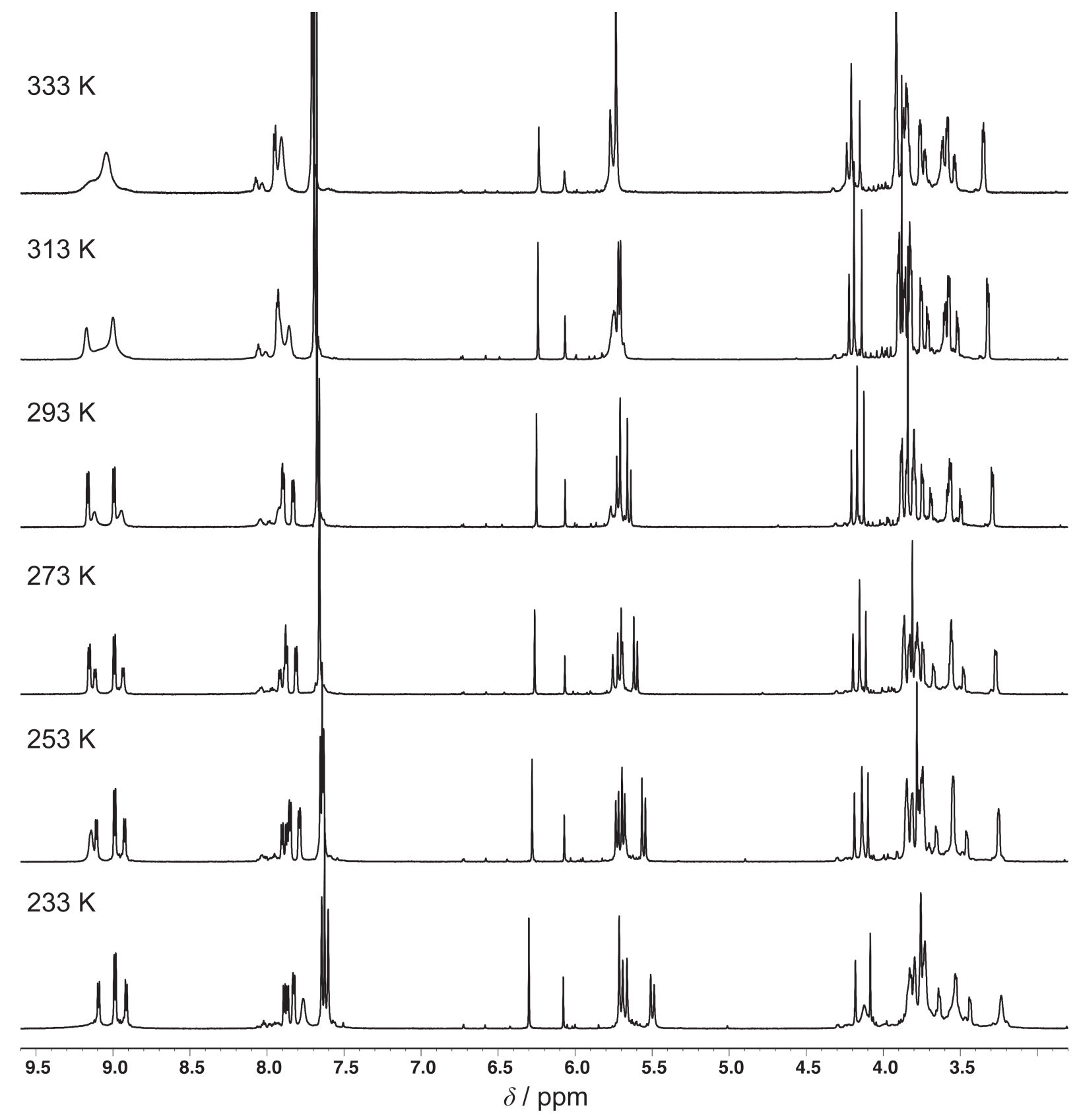

Figure S7: Variable Temperature ${ }^{1} \mathrm{H}$ NMR spectra of 1.4PF $(600 \mathrm{MHz})$ 


\section{Additional Electrochemical Characterization}

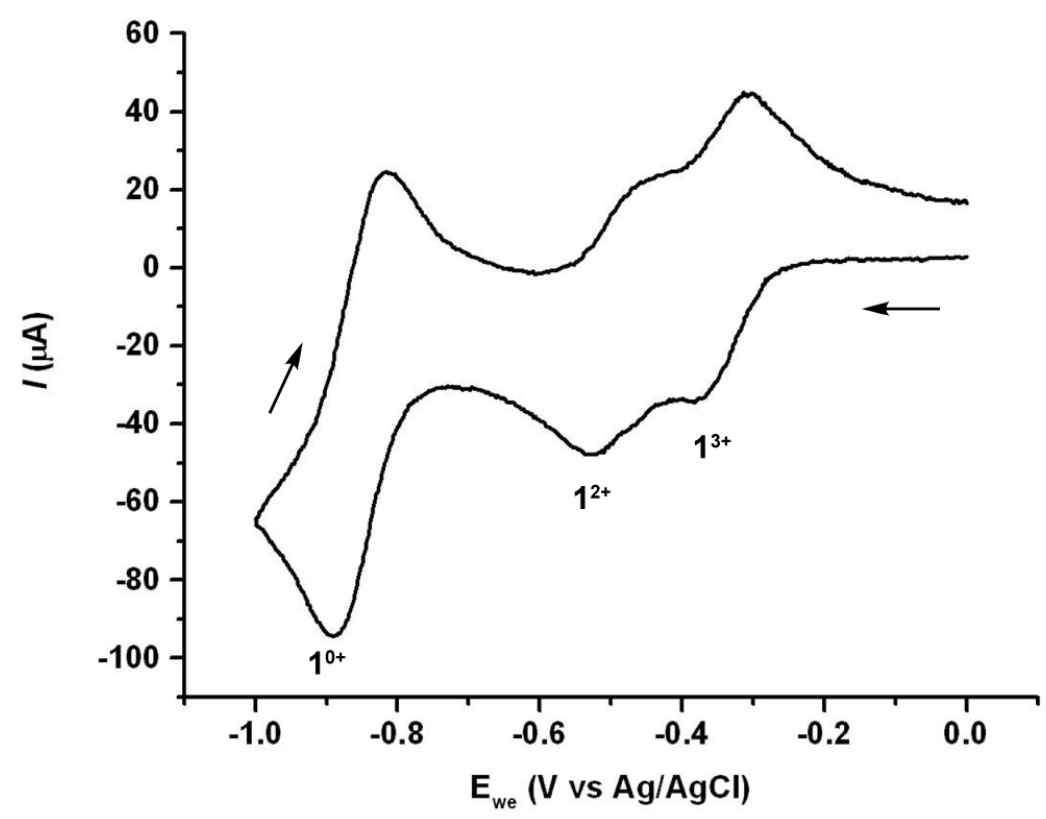

Figure S8: Reductive CV $(200 \mathrm{mV} / \mathrm{s})$ of $\mathbf{1} \cdot 4 \mathrm{PF}_{6}$; reduction of $\mathbf{1}^{4+}$ displaying three reversible processes corresponding to the distinct first and second one electron reductions of the $\mathbf{C B P Q T}^{\mathbf{}}$ ring followed by the two electron process to the fully reduced, neutral $\mathrm{CBPQT}^{4+}$ ring. 


\section{XYZ Coordinates for Calculated Structures}

\begin{tabular}{|c|c|c|c|}
\hline \multicolumn{4}{|c|}{ 1.4PF 6 GSCC } \\
\hline N1 & 0.1257769724 & -0.1159349357 & -0.0708961535 \\
\hline $\mathrm{C} 2$ & 0.0957044104 & -0.1119402762 & 1.2745020165 \\
\hline C3 & 1.2726130830 & -0.1268357485 & 1.9947802425 \\
\hline C4 & 2.5021054036 & -0.1738938441 & 1.3412487632 \\
\hline C5 & 2.4945380310 & -0.2139346909 & -0.0630960404 \\
\hline C6 & 1.3008747947 & -0.1663402256 & -0.7363704205 \\
\hline C7 & 3.7495791467 & -0.1096525667 & 2.1110558215 \\
\hline C8 & 3.7603505780 & -0.4403444664 & 3.4771482699 \\
\hline C9 & 4.9069463933 & -0.2524545479 & 4.2065647992 \\
\hline N10 & 6.0272027331 & 0.2385802054 & 3.6344802877 \\
\hline C11 & 6.0620277797 & 0.5234636746 & 2.3192159043 \\
\hline C12 & 4.9425040167 & 0.3417935460 & 1.5369673054 \\
\hline C13 & 7.1844579309 & 0.6346428095 & 4.4782959445 \\
\hline C14 & 7.1033808844 & 2.0978987779 & 4.8165243668 \\
\hline C15 & 6.2883688970 & 17281 & 6226696 \\
\hline C16 & 6.1070038569 & 3.8872325483 & 6.1052583338 \\
\hline C17 & 6.7658345755 & 4.8222472609 & 5.3078866660 \\
\hline C18 & 7.6341686683 & 4.3953088199 & 4.3080482897 \\
\hline C19 & 7.7800569569 & 3.0411242908 & 4.0418887924 \\
\hline C20 & 6.4223918720 & 6.2843131773 & 5.4090863351 \\
\hline N21 & 5.2915941745 & 6.5673169289 & 4.4736540698 \\
\hline $\mathrm{C} 22$ & 4.0364018300 & 6.2720381401 & 4.8748596177 \\
\hline $\mathrm{C} 23$ & 2.9905351088 & 6.3289761343 & 3.9847958718 \\
\hline C24 & 3.2146031158 & 6.6419923894 & 43038513 \\
\hline $\mathrm{C} 25$ & 4.5372827907 & 0248 & 3756 \\
\hline $\mathrm{C} 26$ & 5.548936 & 044 & 616 \\
\hline C27 & 2.1193005006 & 6.5768503229 & 1.6654399940 \\
\hline $\mathrm{C} 28$ & 0.7720850747 & 6.5943642459 & 2.0880987052 \\
\hline C29 & -0.2436022219 & 6.4315551099 & 1.1869224648 \\
\hline N30 & 0.0089587722 & 6.2460317715 & -0.1337268918 \\
\hline C31 & 1.2851447255 & 6.2291936834 & -0.5790730849 \\
\hline C32 & 2.3390432078 & 6.4241487934 & 0.2888663856 \\
\hline C33 & -1.0993431532 & 5.8731865138 & -1.0562709776 \\
\hline C34 & -1.2980148724 & 4.3800748386 & -1.0688176774 \\
\hline C35 & -1.7964980410 & 3.71 & 42010 \\
\hline C36 & -1.81573 & 2.3 & 2704 \\
\hline C37 & -1.3294841842 & 1.5908138205 & 5338695 \\
\hline C38 & -0.8993840011 & 2.2534585605 & -2.1323927020 \\
\hline C39 & -0.8934875438 & 3.6438976635 & -2.1768304017 \\
\hline C40 & -1.1356191793 & 0.1098866562 & -0.8242568235 \\
\hline $\mathrm{H} 41$ & -0.8807753064 & -0.0891724757 & 1.7616927758 \\
\hline $\mathrm{H} 42$ & 1.1888678269 & -0.0958790093 & 3.0759581541 \\
\hline $\mathrm{H} 43$ & 3.4137687801 & -0.2671933370 & -0.6387633898 \\
\hline $\mathrm{H} 44$ & 1.2396933932 & -0.1448659668 & -1.8203747714 \\
\hline H45 & 2.88714 & -0.8 & 3.9 \\
\hline $\mathrm{H} 46$ & 4.9632210872 & -0.4747213993 & 25861 \\
\hline $\mathrm{H} 47$ & 6.9886555847 & 0.9436518229 & 1.9296781913 \\
\hline $\mathrm{H} 48$ & 5.0075953384 & 0.6509629111 & 0.4966865707 \\
\hline $\mathrm{H} 49$ & 7.1463175638 & -0.0035977458 & 8349767 \\
\hline $\mathrm{H} 50$ & 8.0780708728 & 0.3664477896 & 3.9044908024 \\
\hline H51 & 5.7565200633 & 1.8122908212 & 6.4889269930 \\
\hline H52 & 5.4274382185 & 4.2085366998 & 6.8914550671 \\
\hline H53 & 8.1648425820 & 5.1184845990 & 3.7003743056 \\
\hline H54 & 8.3924816474 & 2.7276449680 & 3.1949476578 \\
\hline H55 & 7.2556375965 & 6.9254719293 & 5.1057743305 \\
\hline H56 & 6.0831385231 & 6.5630001477 & 6.4118017428 \\
\hline $\mathrm{H} 57$ & 3.8937783569 & 5.9636301373 & 5.9096736983 \\
\hline H58 & 2.0185354694 & 6.0418352695 & 4.3755475071 \\
\hline H59 & 4.8185847714 & 7.2068375553 & 1.2412820650 \\
\hline $\mathrm{H} 60$ & 6.5841499298 & 7.1280988271 & 2.9431291233 \\
\hline $\mathrm{H} 61$ & 0.5111385569 & 6.7510549761 & 3.1293464194 \\
\hline
\end{tabular}




\begin{tabular}{|c|c|c|c|}
\hline H62 & -1.2885817525 & 6.4497774210 & 1.4797901396 \\
\hline $\mathrm{H} 63$ & 1.4376652550 & 6.0228748023 & -1.6364405273 \\
\hline & 3.3377913017 & 6.3706567742 & -0.1377466956 \\
\hline & -0.8235737880 & 6.2383624056 & -2.0500006916 \\
\hline H66 & -1.9877015843 & 6.4164890015 & -0.7143135812 \\
\hline H67 & -2.1441176054 & 4.2674371719 & 0.9266492012 \\
\hline & -2.1633631581 & 1.8114154637 & 0.9865143736 \\
\hline & -0.5157077370 & 1.6999957643 & -2.9894271386 \\
\hline $\mathrm{H} 70$ & -0.5031070578 & 4.1491921096 & -3.0545941235 \\
\hline H71 & -1.9289718709 & -0.3662643905 & -0.2412358367 \\
\hline & -1.0455124182 & -0.4152840245 & \\
\hline & -1.7724215444 & & \\
\hline C74 & -3.1366538705 & 1.8091923734 & \\
\hline & -3.4067853319 & 0.3355417327 & 3835011 \\
\hline 076 & -3.1310280696 & -0.3155772468 & \\
\hline $\mathrm{C} 77$ & -3.3734386896 & -1.7030744714 & 2.6057627797 \\
\hline C78 & -3.2009970241 & -2.3527572220 & 1.2553989326 \\
\hline 079 & -1.8478602370 & -2.4795485216 & 0.8718618811 \\
\hline $\mathrm{C} 80$ & -1.2260286122 & -3.6538520661 & 1.3732165162 \\
\hline C81 & 0.1897836066 & -3.3548692760 & 1.7739732926 \\
\hline 082 & 0.96105381 & -2.9720540465 & 0.63 \\
\hline $\mathrm{C} 83$ & 2.04 & -3.816 & 0.3 \\
\hline $\mathrm{C} 84$ & 7.52 & -3.5 & \\
\hline 085 & & -2.27 & \\
\hline C86 & 9.430 & -2.41 & 4.85 \\
\hline C87 & 10.0739972116 & 344389 & 4585946 \\
\hline 088 & 10.1880439879 & -0.4857308274 & 3.7623733755 \\
\hline C89 & 10.6312243823 & 0.8490449153 & 3.7730494122 \\
\hline $\mathrm{C9O}$ & 10.6558453578 & 1.3479042 & 2.3503750189 \\
\hline 091 & 9.39474147 & 1.13810122 & 1.7543688346 \\
\hline C92 & 9.38703716 & 1.3689959363 & 0.3559650920 \\
\hline $\mathrm{C} 93$ & 8.72 & & -0.0 \\
\hline 094 & 7.32 & & \\
\hline C95 & 6.57 & $3.4 \varepsilon$ & -0.5 \\
\hline C96 & 5.17 & 3.44 & -0.0 \\
\hline C97 & 4.0858416197 & 3.2976868401 & 352879 \\
\hline S98 & 2.5151388792 & 3.2717050393 & 3064270 \\
\hline C99 & 3.1764497782 & 3.3439066716 & 2074858 \\
\hline S100 & 4.90980431 & & 576025 \\
\hline & 2.3753030408 & 3.1757127673 & 2.6807182091 \\
\hline S10 & 2.9878980062 & 3.0422655 & 8668212 \\
\hline & & & \\
\hline & & 2.5 & 4.1 \\
\hline & 0.6 & 3.0 & \\
\hline & 30 & 2.1 & 4.56 \\
\hline & -3.672 & 2.187 & 8466877 \\
\hline & -3.5240468523 & 2.3457170320 & 4.4437235051 \\
\hline & -2.8044801067 & -0.0995597091 & 4.5246778429 \\
\hline & -4.4717632842 & 0.1983447962 & 32609550 \\
\hline & -4.4088575273 & -1.8704629543 & 8344631 \\
\hline & -2.6910470335 & -2.1484441 & 9488032 \\
\hline & & & \\
\hline & -3.70 & -1.73 & 0.4 \\
\hline & -1.25 & -4.443 & 0.60 \\
\hline & -1.75100 & -4.025 & 0237350 \\
\hline & 0.188 & -2.5389952775 & 0554582 \\
\hline $\mathrm{H} 118$ & 0.6322910612 & -4.2254788402 & 2.2792754417 \\
\hline $\mathrm{H} 119$ & 2.3513907311 & -3.5920296573 & -0.6830427097 \\
\hline & 1.7342421336 & -4.8787317288 & 0.3557214342 \\
\hline & 7.3205332399 & -4.0464945864 & 5.1498345055 \\
\hline & 8.2299141739 & -4.1962795531 & 3.6401356935 \\
\hline & 10.0278859093 & -3.0178332547 & 4.1869770992 \\
\hline & 9.4377418643 & -2.9300544789 & 5.8654677821 \\
\hline & 9.4753987734 & -0.4175332673 & 5.7073648562 \\
\hline
\end{tabular}




\begin{tabular}{|c|c|c|c|}
\hline H126 & 11.0632986542 & -1.1811975247 & 5.5100466449 \\
\hline $\mathrm{H} 127$ & 11.6462610268 & 0.9326532414 & 4.1993813924 \\
\hline $\mathrm{H} 128$ & 9670515019 & 1.4730747761 & 4.3980663645 \\
\hline $\mathrm{H} 129$ & 10.9306494134 & 2.4183676634 & 2.3400502130 \\
\hline $\mathrm{H} 130$ & 11.4270207098 & 0.7936635103 & 1.7888494591 \\
\hline H131 & 8.8382691247 & 0.5396382941 & -0.1127103377 \\
\hline $\mathrm{H} 132$ & 10.4139942000 & 1.3530017245 & -0.038433567 \\
\hline $\mathrm{H} 133$ & 9.1002665139 & 3.5091214461 & 0.5742680791 \\
\hline $\mathrm{H} 134$ & 8.9365348998 & 2.8836224309 & -1.0809639209 \\
\hline $\mathrm{H} 135$ & 6.5803742916 & 3.2669308099 & -1.6241094834 \\
\hline $\mathrm{H} 13$ & 7.0159201766 & 4.4795363134 & \\
\hline $\mathrm{H} 13$ & 4.0947181412 & 3.2360174719 & \\
\hline $\mathrm{H} 138$ & 1.4263586303 & 2.3845785649 & 6.064 \\
\hline $\mathrm{H} 139$ & -0.9990700986 & 1.2219605938 & 5.0915287190 \\
\hline $\mathrm{H} 140$ & -1.4682238682 & 2.9375688572 & \\
\hline P141 & 2.3561853736 & 4.5751734091 & -3.8706206647 \\
\hline F142 & 2.8552132203 & 5.5616193649 & -5.0171773272 \\
\hline F143 & 3.1870066176 & 5.4192246496 & -2.7390675022 \\
\hline F144 & 1.0567897339 & 5.5576072714 & -3.5717095541 \\
\hline F145 & 1.4608047674 & 3.7252888454 & -4.8995217114 \\
\hline F146 & 3.6247467341 & 3.5957207617 & -4.0738651845 \\
\hline F147 & 4117641 & 3.587 & -2.6 \\
\hline P148 & 8.667 & 6.45 & \\
\hline F149 & 9.76 & 7.56 & \\
\hline F150 & & 7.0108759567 & 2.75 \\
\hline F151 & 7.4603807731 & 7.4627414270 & 0.7922511081 \\
\hline F152 & 8.7311045251 & 5.8681301404 & -0.2966568306 \\
\hline F153 & 9.8014322546 & 5.4000727691 & 1.6909715473 \\
\hline F154 & 7.5231236024 & 5.3262420807 & 1.5782280145 \\
\hline P155 & -0.0658313536 & -1.6476170842 & \\
\hline F156 & -0.6517356943 & -0.8673346725 & \\
\hline F157 & & -0.277 & \\
\hline F158 & & -2.088 & \\
\hline F159 & -0.8 & -2.9 & 4.7 \\
\hline F160 & -1.37 & -1.15 & 6.0 \\
\hline F161 & 0.5142516344 & -2.399 & \\
\hline P162 & 2.5270278959 & 4.4920595647 & 8.00 \\
\hline F163 & 3.1231396396 & 3.8980590541 & 9.36 \\
\hline F164 & 1.7543331055 & 5.6573126470 & \\
\hline F165 & 3.8521541044 & $5.4671627 \varepsilon$ & \\
\hline F166 & 3.3414901403 & 3.3388162 & \\
\hline F167 & & & \\
\hline F168 & 1.9 & 5.0 & \\
\hline C169 & & -3.4 & \\
\hline C1 & 5.28 & -3.4 & 2.7 \\
\hline C171 & 4.16 & -3.555 & 636098 \\
\hline C172 & 3.1894684934 & -3.653 & 1.24 \\
\hline \multicolumn{4}{|c|}{ 1.4PF 6 MSCC } \\
\hline $\mathrm{N} 1$ & -0.5316580074 & & 3479090 \\
\hline $\mathrm{C} 2$ & 580708 & 383685 & 1.4060319883 \\
\hline $\mathrm{C} 3$ & & 73 & 2.02 \\
\hline C4 & & 96880 & 1.256 \\
\hline C5 & 20 & 42 & -0.128 \\
\hline C6 & & -0.24 & -0.694 \\
\hline $\mathrm{C} 7$ & 3.2897488471 & 0.1179036475 & 4346540 \\
\hline $\mathrm{C} 8$ & 3.4285594098 & 0.2041762869 & 3.2547719518 \\
\hline $\mathrm{Cg}$ & 4.6372916128 & 0.5332717489 & 3.8126117150 \\
\hline N10 & 5.7164679650 & 0.7514075913 & 3.0310107625 \\
\hline C11 & 5.6286213876 & 0.6407025990 & 1.6902041127 \\
\hline $\mathrm{C} 12$ & 4.4324976859 & 0.3286474158 & 1.0850439302 \\
\hline $\mathrm{C} 13$ & 6.9593697089 & 1.3021380231 & 3.6294864619 \\
\hline C14 & & 2.8033577715 & 3.5533529616 \\
\hline & 6.2357709715 & 3.5416124832 & 4.4976388732 \\
\hline
\end{tabular}




\begin{tabular}{|c|c|c|c|}
\hline 16 & 6.1450877592 & 4.9209469793 & 4.3778536637 \\
\hline C17 & 6.7542689142 & 5.5665969229 & 3.3044420923 \\
\hline C18 & 7.4670010773 & 4.8320488763 & 2.3545986832 \\
\hline C19 & 7.5629494364 & 3.4536766143 & 2.4835707586 \\
\hline 20 & 6.5169582442 & 7.0376055826 & 3.0932116208 \\
\hline N21 & 5.4259886061 & 7.1909332573 & 2.0862784645 \\
\hline $\mathrm{C} 22$ & 4.1607549815 & 6.9779331972 & 2.5011833026 \\
\hline $\mathrm{C} 23$ & 3.1450888772 & 6.8279349206 & 1.5830224639 \\
\hline $\mathrm{C} 24$ & 3.4511624944 & 6.8840631043 & 0.2233274313 \\
\hline $\mathrm{C} 25$ & 4.7529194896 & 7.1834593733 & -0.1760845967 \\
\hline $\mathrm{C} 26$ & 5.7330792336 & 7.3250855288 & 0.7803460558 \\
\hline $\mathrm{C} 27$ & 2.4383836871 & 6.5345443022 & -0.7854045142 \\
\hline $\mathrm{C} 28$ & 1.1307008169 & 7.0306544873 & -0.7109856988 \\
\hline $\mathrm{C} 29$ & 0.1969086572 & 6.6001917257 & -1.6201562548 \\
\hline N30 & 0.5167158718 & 5.6759102499 & -2.5541237927 \\
\hline C31 & 1.7722854037 & 5.1945259640 & -2.6579909564 \\
\hline C32 & 2.7631390241 & 5.6404531157 & -1.8073909684 \\
\hline C33 & -0.5644832470 & 5.0310720894 & -3.3393315842 \\
\hline C34 & -1.0345506227 & 3.7776651821 & -2.6473433113 \\
\hline C35 & -1.2656356525 & 3.7637317769 & -1.2705568941 \\
\hline C36 & -1.5980839978 & 2.5879767121 & -0.6242645210 \\
\hline C37 & -1.7206700160 & 1.4076149406 & -1.3572490023 \\
\hline C38 & -1.5355451643 & 1.4250176927 & -2.7346841486 \\
\hline C39 & -1.1843319646 & 2.6054133733 & -3.3784705069 \\
\hline C40 & -1.8345655053 & 0.1154937861 & -0.6023139952 \\
\hline H41 & -1.3473383859 & 0.1817463133 & 1.9459109910 \\
\hline $\mathrm{H} 42$ & 0.8066262083 & 0.0816671713 & 3.1072443155 \\
\hline $\mathrm{H} 43$ & 2.6646996460 & -0.2507129452 & -0.8064333161 \\
\hline $\mathrm{H} 44$ & 0.4420117383 & -0.2848112720 & -1.7714802653 \\
\hline $\mathrm{H} 45$ & 2.6028268869 & 0.0561979213 & 3.9394870302 \\
\hline $\mathrm{H} 46$ & 4.7435865593 & 0.6513821175 & 4.8872561033 \\
\hline H47 & 6.5110170432 & 0.8875580021 & 1.1031643264 \\
\hline $\mathrm{H} 48$ & 4.4012749208 & 0.3554209720 & -0.0006778734 \\
\hline $\mathrm{H} 49$ & 7.0029419570 & 0.9427237963 & 4.6619243330 \\
\hline $\mathrm{H} 50$ & 7.8010904718 & 0.8725341819 & 3.0779392115 \\
\hline H51 & 5.7096151053 & 3.0549157856 & 5.3187127348 \\
\hline $\mathrm{H} 52$ & 5.5488693334 & 5.4799027839 & 5.0947421209 \\
\hline $\mathrm{H} 53$ & 7.9239202046 & 5.3252043317 & 1.4944455094 \\
\hline $\mathrm{H} 54$ & 8.0930683483 & 2.8778543039 & 1.7242453770 \\
\hline $\mathrm{H} 55$ & 7.3939068150 & 7.5595726624 & 2.6980302431 \\
\hline $\mathrm{H} 56$ & 6.1828904275 & 7.5301873013 & 4.0113045607 \\
\hline $\mathrm{H} 57$ & 3.9950211071 & 6.8967722379 & 3.5738317763 \\
\hline H58 & & 6.5883486269 & 1.96888880294 \\
\hline $\mathrm{H} 59$ & 5.0429690344 & 7.2611943517 & -1.2185878069 \\
\hline $\mathrm{H} 60$ & 6.7705830300 & 7.4883313968 & 0.5031412426 \\
\hline $\mathrm{H} 61$ & 0.8485350823 & 7.7536199002 & 0.0483523231 \\
\hline $\mathrm{H} 62$ & & 6.9496925758 & -1.6214188261 \\
\hline $\mathrm{H} 63$ & 1.9435224750 & 4.4173948118 & -3.3995230809 \\
\hline $\mathrm{H} 64$ & & & -1.9130162377 \\
\hline $\mathrm{H} 65$ & -0.1609267626 & 4.8010468542 & -4.3304181711 \\
\hline $\mathrm{H} 66$ & -1.3576342686 & 5.7784054167 & -3.4621194669 \\
\hline $\mathrm{H} 67$ & -1.1373384474 & 4.6631384720 & -0.6706618073 \\
\hline $\mathrm{H} 68$ & -1.7005384587 & 2.5726839918 & 0.4616305425 \\
\hline $\mathrm{H} 69$ & -1.6070441486 & 0.5009443009 & -3.3071355603 \\
\hline $\mathrm{H} 70$ & & & -4.4447120457 \\
\hline $\mathrm{H} 71$ & -2.5924793256 & 0.1655954167 & 0.1860587130 \\
\hline $\mathrm{H} 72$ & & -0.7351625797 & -1.2585508106 \\
\hline C73 & 0.7690729257 & 3.3109488413 & 3.1175532193 \\
\hline 074 & -0.5032564592 & 2.8551932374 & 2.6694829435 \\
\hline C75 & -1.3353732507 & 2.6276943489 & 3.7940955107 \\
\hline C76 & & 2.5015815365 & 3.3318767845 \\
\hline 077 & -2.9932850857 & 1.3680217465 & 2.5027490936 \\
\hline C78 & & & 3.1121503754 \\
\hline & 2837517 & -0.4867338606 & 4.10028 \\
\hline
\end{tabular}




\begin{tabular}{|c|c|c|c|}
\hline 80 & 3.4984569990 & -1.7152056497 & 4.2847158710 \\
\hline & & -2.5708152672 & 5.2196570574 \\
\hline & -1.5716688034 & -3.1626149404 & 4.7512228959 \\
\hline & -0.5393354725 & -2.1999411424 & 4.8170455255 \\
\hline & 7.0297679430 & -1.7703360611 & -1.3043535164 \\
\hline & 7589177130 & -2.8836885445 & -1.7702222972 \\
\hline & 9321215556 & -3.0792196544 & -0.8293996764 \\
\hline & .6521179932 & -1.9031551817 & -0.5771419808 \\
\hline & 10.4072051575 & -1.4293473943 & -1.6693186944 \\
\hline & 10.9557472114 & -0.0568163196 & -1.3333375493 \\
\hline & 10.0744806706 & 0.9961532960 & $\begin{array}{l}-1.6201293361\end{array}$ \\
\hline & 8.9194796069 & 1.0393920394 & -0.8185513663 \\
\hline & 8.1423448872 & 2.2664235330 & $\begin{array}{l}-1.2288926533 \\
\end{array}$ \\
\hline & 6.9011447451 & 2.2237126720 & \\
\hline & 6.1118660444 & 3.3551373985 & -0.8784512954 \\
\hline & 1.1391676376 & 2.6841159040 & \\
\hline 36 & 0.6959685455 & 4.3243627624 & 3.5449576519 \\
\hline & -1.2647067326 & 3.4734420589 & \\
\hline 38 & -0.9949719769 & 1.7367767213 & 4.3504147806 \\
\hline & -3.4312722269 & 2.4732090854 & 4.2070167253 \\
\hline 100 & -3.0254276268 & 3.3908974691 & 2.7410882699 \\
\hline & & -0.3889537916 & \\
\hline 102 & -4.6131374910 & 0.6487838783 & 3.5885047749 \\
\hline & -1.8207582142 & -0.6367724940 & 3.7054237965 \\
\hline 102 & -2.7151349735 & 0.0450187762 & 5.0620846938 \\
\hline & & & \\
\hline 106 & -2.7186276437 & -2.0496324316 & 6.1782016530 \\
\hline 10 & -1.3300857403 & -4.0317527255 & 5.3 \\
\hline 108 & -1.6869577408 & -3.5290615413 & 59664824 \\
\hline & & -2.7086408042 & 2852435 \\
\hline 110 & 987257 & 976216 & -1.7681644979 \\
\hline 111 & 44264 & 846802 & \\
\hline 112 & 54562 & 9266244 & -1.2301241832 \\
\hline $\mathrm{H} 113$ & 908060 & 9363237 & 6873204 \\
\hline $\mathrm{H} 114$ & 8933928 & 24977455 & 2943783 \\
\hline $\mathrm{H} 115$ & .4777367 & 35480 & 0966942 \\
\hline H116 & 332122 & 206749 & 3477335 \\
\hline H117 & & 8388636 & 0.2564371552 \\
\hline$H 118$ & & 570885 & 006912 \\
\hline H119 & & 4253453 & -0.98 \\
\hline $\mathrm{H} 120$ & & 4571 & 3138 \\
\hline $\mathrm{H} 121$ & & & \\
\hline P122 & & & \\
\hline $\mathrm{F} 123$ & & & \\
\hline $\mathrm{F} 124$ & & & \\
\hline F125 & & & \\
\hline F126 & & & \\
\hline F127 & & & \\
\hline F128 & & & -1.9 \\
\hline P129 & & & \\
\hline $\mathrm{F} 130$ & & & \\
\hline F131 & & & \\
\hline F132 & & & \\
\hline F133 & & & \\
\hline F134 & & & \\
\hline F135 & & 5.9034168056 & -2.35 \\
\hline P136 & & 69706139 & \\
\hline $\mathrm{F} 13$ & & & \\
\hline F138 & & 2.0749403 & 20210? \\
\hline F139 & & 0.4146534 & 5.09 \\
\hline $\mathrm{F} 140$ & & & 7.3664723248 \\
\hline $\mathrm{F} 14$ & & 1.7735400015 & 7.6817772387 \\
\hline F142 & & -0.0022590568 & 6.271252230 \\
\hline P143 & 2.5649051641 & 5.6990171977 & 5.550571882 \\
\hline
\end{tabular}




$\begin{array}{llll}\text { F144 } & 3.4475599533 & 4.7897551972 & 6.5345059295 \\ \text { F145 } & 2.0103688635 & 6.5894718242 & 6.7570560197 \\ \text { F146 } & 3.8569460221 & 6.7493506472 & 5.4069665509 \\ \text { F147 } & 3.1613400438 & 4.8683533875 & 4.2681522006 \\ \text { F148 } & 1.3102569714 & 4.7031632816 & 5.6401925363 \\ \text { F149 } & 1.7607892288 & 6.6373366068 & 4.4626557453 \\ \text { C150 } & 5.7636675633 & -1.5981463507 & -1.8860877278 \\ \text { H151 } & 5.5320139006 & -0.5246877326 & -1.8178893895 \\ \text { H152 } & 5.7591317671 & -1.8579197026 & -2.9573330046 \\ \text { C153 } & 0.6600376780 & -2.5802662402 & 5.4463014358 \\ \text { H154 } & 0.4976479596 & -3.4913501087 & 6.0468359692 \\ \text { C155 } & 3.4835648444 & -2.6983697788 & -1.5365681606 \\ \text { C156 } & 4.7234913428 & -2.3907337720 & -1.1421291961 \\ \text { S157 } & 5.1732759697 & -2.8459189838 & 0.5135700895 \\ \text { C158 } & 3.5014395505 & -3.1948135295 & 0.9913498593 \\ \text { S159 } & 2.4077873869 & -3.4776166895 & -0.3729827603 \\ \text { C160 } & 3.1182708718 & -3.2248417321 & 2.2937653595 \\ \text { S161 } & 4.2961359431 & -3.0501703254 & 3.6038584943 \\ \text { C162 } & 3.0770218965 & -2.6463021200 & 4.8007091082 \\ \text { C163 } & 1.7900352179 & -2.8133561348 & 4.4848101384 \\ \text { S164 } & 1.4416850306 & -3.4081808995 & 2.8457161887 \\ \text { H165 } & 3.0770716928 & -2.4611327928 & -2.5172093473 \\ \text { H166 } & 0.9619164367 & -1.7756204726 & 6.1299353378 \\ \text { H167 } & 3.4090769525 & -2.2357079979 & 5.7499119239 \\ \text { H168 } & 6.6795298206 & 4.2824134915 & -0.7033905760 \\ \text { C169 } & 1.7704503758 & 3.3088711710 & 2.0714812609 \\ \text { C170 } & 2.7406605147 & 3.3654805711 & 1.3425465865 \\ \text { C171 } & 3.8710404413 & 3.3943016158 & 0.5704126773 \\ \text { C172 } & 4.8908820090 & 3.3769430830 & -0.0931960226\end{array}$

